\title{
PARTICIPAÇÃO DO USUÁRIO EM PROJETOS ABERTOS DE TECNOLOGIA ASSISTIVA
}

\section{USER PARTICIPATION IN OPEN ASSISTIVE TECHNOLOGY PROJECTS}

\author{
Graziela Guzi de Moraes ${ }^{1}$, D.Eng. \\ grazzi.guzzi@gmail.com e https://orcid.org/0000-0003-0454-5510 \\ Giovana Mara Zugliani Bortolan ${ }^{1}$, M. Sc. \\ gmzugliani@gmail.com e https://orcid.org/0000-0002-2000-9542 \\ Yoshimasa Sagawa Jr. ${ }^{2}$, D. Sc. \\ sagawajunior@gmail.com e https://orcid.org/0000-0002-1024-3095 \\ Susana Cristina Domenech ${ }^{1}$, D.Eng. \\ scdomenech@gmail.com e https://orcid.org/0000-0001-7517-0723 \\ Marcelo Gitirana Gomes Ferreira ${ }^{1}$, D.Eng. \\ marcelo.gitirana@gmail.com e https://orcid.org/0000-0003-1912-9982
}
${ }^{1}$ Laboratório de Interfaces e Interações em Tecnologias Assistivas (Li2TA), Universidade do Estado de Santa Catarina (UDESC), Florianópolis, Brasil.
${ }^{2}$ Laboratório do Movimento/Hospital Universitário, Besançon, França.

código aberto, plataforma colaborativa, tecnologia assistiva, design centrado no usuário As plataformas Open-Source têm permitido a participação dos usuários no desenvolvimento de produtos e serviços. Sendo assim, esta pesquisa objetivou gerar conhecimento teórico para subsidiar o desenvolvimento de plataformas colaborativas de disponibilização aberta de tecnologia assistiva. A revisão bibliográfica sistemática buscou a definição do conceito de código aberto, design participativo e design centrado no usuário, bem como suas demais nomenclaturas e variações. Os resultados apresentam uma comparação do design centrado no humano, do design participativo e do design aberto e analisa como ocorre a participação do usuário no desenvolvimento de ferramentas e plataformas abertas, de modo a propor soluções de projetos para dispositivos médicos em tecnologia assistiva.

open source, collaborative platform, assistive technology, user centered design As Open-Source platforms, they have allowed the participation of users in the development of products and services. This research aimed to generate theoretical knowledge to support the development of collaborative platforms and the open availability of assistive technology. A systematic literature review sought to define the concept of open source, participatory design, user centred design, as well as its other nomenclatures and variations. The results present a discussion of how user participation occurs in the development of tools and open platforms, to project proportions for medical devices in assistive technology.

Recebido em: $08 / 09 / 2020$

Aceito em: 26 / 06 / 2021

DOI: http://dx.doi.org/10.22570/ergodesignhci.v9i1.1470 


\section{Introdução}

A tecnologia assistiva (TA) se refere a uma "ampla gama de dispositivos, serviços, estratégias e práticas que são concebidas e aplicadas para melhorar os problemas enfrentados por pessoas que têm deficiência", proporcionando independência, qualidade de vida e inclusão social (COOK e POLGAR, 2008, p. 5). As TAs ampliam a comunicação, a mobilidade, o controle do ambiente, as habilidades de aprendizado, o trabalho e a integração na vida familiar, entre os usuários com seus familiares, amigos e a sociedade em geral (BERSCH, 2013). Portanto, a aplicação da TA abarca uma série de possibilidades do desempenho humano, desde tarefas básicas de autocuidado (mobilidade, comunicação, manutenção do lar, preparo de alimentos, tarefas ocupacionais), até atividades de lazer e de trabalho.

Um conceito empregado na área da Ergonomia e dos Fatores Humanos para criar e adaptar produtos e sistemas para os usuários, com base em suas capacidades físicas e psicológicas, é o design centrado no usuário (DCU). Segundo a Interaction Design Foundation (2021, sem página), o design centrado no usuário "é um processo de design iterativo no qual os designers se concentram nos usuários e em suas necessidades em cada fase do processo". O objetivo da utilização de uma metodologia desta natureza, é desenvolver empatia e entender os potenciais usuários.

Neste contexto, Lanutti et al. (2015) argumentam que existem aspectos subjetivos que influenciam a percepção dos usuários sobre a experiência no uso de dispositivos de tecnologia assistiva. Sendo assim, outro conceito de maior abrangência, com aspectos semelhantes, porém ampliados, começou a ser utilizado nas pesquisas: o Design Centrado no Humano (DCH). O DCH, foi formalizado pela norma ISO 9241-210 (2010), e trata-se de uma abordagem menos racional e mais analítica, ou seja, menos interessado em atribuir funções aos objetos (funcionalidade e usabilidade) e mais interessado em compreender os significados que os produtos têm para os usuários, buscando incluir o design da experiência, o desejo, o prazer e os fatores emocionais (PEZZINI; SCHULENBURG; ELY, 2018; CHAVES, 2019). Para McDonagh et al. (2021), o design centrado no humano aprecia soluções de dispositivos de tecnologia assistiva que incluem as necessidades funcionais, emocionais, sociais e culturais dos usuários. O desenvolvimento de soluções sem considerar a tomada de decisão das necessidades funcionais e subjetivas pode levar à baixa aceitação dos produtos e serviços de TA.

O Design Participativo surgiu por meio de equipes interdisciplinares para atender e executar atividades projetuais cada vez mais complexas, com múltiplos requisitos e curto espaço de tempo (FONTANA; HEEMANN; FERREIRA, 2012). O processo perpassa todos os níveis de trabalho: estratégico (definição de problemas e objetivos), tático (abordam as metas pelas quais o objetivo deve ser alcançado) e operacional (o que de fato será realizado). Assim, o design participativo demanda um alto nível de confiança e comprometimento entre os envolvidos (HEEMANN; LIMA; CORRÊA, 2010).

Os processos abertos (open sources) incentivam a participação dos usuários como designers cidadãos. O termo aplicado por Hamidi et al. (2014), designa pessoas que não são designers profissionais (ou seja, não têm treinamento formal em design e não trabalham com projetos), mas criam métodos e os usam para encontrar soluções para os problemas do mundo real. Esses designers amadores, usam métodos de impressão 3D, porém para a produção de TA ainda é um processo pouco desenvolvido, pois estes possuem barreiras de conhecimento limitado em programação e eletrônica, barreiras de custos e barreiras físicas e cognitivas. Hurst e Kane (2013) já haviam identificado a necessidade de desenvolver ferramentas para tornar o "fazer" mais acessível.

O ambiente de participação, que pode ser físico ou digital, depende do acesso dos usuários às tecnologias, treinamento ou, em alguns casos, de mediação. As ferramentas são os instrumentos de participação dos usuários, que incluem métodos de comunicação de grupos e o uso das ferramentas, como softwares por exemplo. A interação do usuário com as ferramentas ocorre por meio dos boundary objects. Segundo Star e Griesemer (1989) boundary objects são ferramentas com as quais, pessoas diferentes interagem quando 
trabalham em torno de um interesse comum, por exemplo: esboços, maquetes, protótipos, mapas conceituais, arquivos relacionados ao design de hardware (contendo as imagens, esquemas e descrições do hardware), entre outros.

À vista disso, envolver os usuários em um processo de design colaborativo, auxilia a organização das ideias do usuário final e desperta a criatividade. Arias, Hurst e Perr (2020) explicam que, pessoas com deficiência e os profissionais relacionados à assistência destes, possuem muitas ideias para a produção de TAs voltadas para tornarem estes usuários mais independentes. No entanto, segundo Soares et al. (2019), a dinâmica colaborativa incide em uma série de restrições e problemas, como questões de comunicação, econômicas e de tempo, bem como a análise de custo/benefício realizada pela empresa no que diz respeito à seleção desses processos. Os autores explicam que há a necessidade de mais pesquisas que incluam a interação do usuário no processo de desenvolvimento de TAs, "a fim de encontrar os benefícios reais do processo, descrevendo todas as situações complexas encontradas pela organização (...) além dos impactos (positivos e negativos) no processo formativo do usuário em relação à imersão" (SOARES et al., 2019, p. 7).

Dessa forma, fica evidente a importância da disponibilização de projeto de tecnologia assistiva, desenvolvidas por meio de processos colaborativos, de forma aberta, para aqueles que necessitam, como usuários; profissionais da saúde, como fisioterapeutas e terapeutas ocupacionais; e familiares. Assim, este trabalho tem por objetivo investigar a participação dos usuários de TA em processos abertos.

\section{Metodologia}

Por meio de uma revisão bibliográfica sistemática (RBS), buscou-se a compreensão dos conceitos relacionados ao Open Source (código aberto), Open Technology (tecnologia aberta), Co-design e Design Colaborativo, bem como suas demais nomenclaturas e variações; os estágios de desenvolvimento e os ambientes e as ferramentas necessárias para a participação do usuário.

O processo de revisão seguiu o protocolo elaborado por Ferenhof e Fernandes (2016):

- Estratégia de busca: foram utilizados os operadores lógicos (user AND open design);

- Consulta em base de dados: a pesquisa foi realizada na base de dados Scopus;

- Padronização e seleção dos documentos: foram estabelecidos alguns critérios de seleção. Primeiramente, selecionadas as principais palavras-chave, idiomas, selecionadas as áreas de conhecimento. Assim, realizou-se a leitura dos títulos levando à escolha daqueles que se alinhavam ao tema da busca para posterior leitura dos resumos;

- Portfólio de documentos: leitura dos artigos, permitindo, em sequência, mais uma filtragem para excluir os que não demonstraram aderência ao tema investigado e os que não obtinham acesso gratuito.

As buscas foram atualizadas em junho de 2021, e não foram limitadas por data ou ano de publicação. A busca atualizada gerou 16.723 documentos. Foram aplicados filtros para área de conhecimento de ciências sociais aplicadas, artigos e revisões de periódicos, totalizando em 1549 documentos. Neste momento foram selecionados somente periódicos acadêmicos que continham as seguintes palavras-chaves: "open systems", "user-computer interface", "open source", “ user-centered design", "user centered design", "open innovation", "participatory design" e "open sources" nos idiomas inglês e português.

Posteriormente, na etapa de extração, foi realizada a leitura dos resumos de 164 artigos. Nesta etapa, foram excluídos 109, que estavam relacionados a pesquisas médicas, métodos de aprendizagem, ou uso de serviços ou ambientes. Restaram 55 artigos, sendo realizada a leitura completa somente dos 42 disponíveis. Em seguida, o portifólio foi finalizado com 4 artigos (Tabela 1). 
Tabela 01. Artigos selecionados na revisão sistemática. Fonte: Elaborado pelos autores (2021).

\begin{tabular}{|c|c|c|c|}
\hline Autores/Ano & Open/Objetivos & $\begin{array}{l}\text { Usuário/ } \\
\text { Ferramenta/Ambiente }\end{array}$ & Participação \\
\hline (LEBER et.al. 2018) & $\begin{array}{l}\text { Inovação aberta/ O objetivo } \\
\text { foi responder se um } \\
\text { comprador (cliente / } \\
\text { consumidor) pode participar } \\
\text { ativamente do processo de } \\
\text { desenvolvimento de um } \\
\text { novo produto. }\end{array}$ & $\begin{array}{l}\text { Usuário Final / } \\
\text { Questionário }\end{array}$ & $\begin{array}{l}\text { Usuário participa nos } \\
\text { estágios iniciais do } \\
\text { processo, em que o processo } \\
\text { é abstrato e os custos são } \\
\text { menores pode ser grande } \\
\text { contribuição para o sucesso } \\
\text { do produto. }\end{array}$ \\
\hline (GEORGES, ET. AL. 2015) & $\begin{array}{l}\text { Inovação aberta/ O objetivo } \\
\text { deste artigo é refletir sobre } \\
\text { os fatores que } \\
\text { desempenham um papel no } \\
\text { envolvimento dos usuários } \\
\text { finais para participarem dos } \\
\text { testes de campo do Living } \\
\text { Lab. }\end{array}$ & $\begin{array}{l}\text { Usuário Final/ } \\
\text { Workshop (ambiente: } \\
\text { living lab) e } \\
\text { questionários online) }\end{array}$ & $\begin{array}{l}\text { Usuário participa em } \\
\text { estágio final ou de } \\
\text { maturidade funcional } \\
\text { percebida da inovação e o } \\
\text { grau em que um protótipo } \\
\text { se assemelha ao produto } \\
\text { final. }\end{array}$ \\
\hline (SPAN et.al., 2014) & $\begin{array}{l}\text { Design participativo/ O } \\
\text { objetivo do estudo foi } \\
\text { avaliar a facilidade de uso } \\
\text { do ferramenta interativa } \\
\text { (DecideGuide), aceitação e } \\
\text { satisfação do usuário e a } \\
\text { opinião dos participantes } \\
\text { para a tomada de decisões. }\end{array}$ & $\begin{array}{l}\text { Usuários finais (Pessoas } \\
\text { com demência, } \\
\text { cuidadores informais e } \\
\text { especialistas)/(focus } \\
\text { group, testes de } \\
\text { usabilidade, } \\
\text { competências } \\
\text { cognitivas) }\end{array}$ & $\begin{array}{l}\text { Os usuários participam no } \\
\text { início e no final do projeto } \\
\text { (feedback). O envolvimento } \\
\text { de todos os usuários finais, } \\
\text { foi importante no } \\
\text { desenvolvimento de uma } \\
\text { ferramenta útil e amigável. }\end{array}$ \\
\hline (MARTIN et. al. 2012) & $\begin{array}{l}\text { Design centrado no } \\
\text { humano/ O objetivo deste } \\
\text { estudo foi investigar os } \\
\text { requisitos do usuário para } \\
\text { um dispositivo médico } \\
\text { (imagem). }\end{array}$ & $\begin{array}{l}\text { Usuários Finais/ } \\
\text { Entrevistas } \\
\text { semiestruturadas }\end{array}$ & $\begin{array}{l}\text { Os usuários participam } \\
\text { durante os estágios iniciais } \\
\text { de desenvolvimento de } \\
\text { dispositivos médicos. } \\
\text { A consulta inicial } \\
\text { identificou uma necessidade } \\
\text { clínica para o dispositivo, as } \\
\text { populações-alvo definidas e } \\
\text { seus requisitos coletados. } \\
\text { Os altos riscos e custos de } \\
\text { desenvolvimento de um } \\
\text { novo dispositivo médico } \\
\text { tornam essencial que seja } \\
\text { seguro e melhore a saúde } \\
\text { dos pacientes. }\end{array}$ \\
\hline
\end{tabular}

A leitura destes trabalhos foi correlacionada com pesquisa assistemática exploratória e buscou responder às seguintes questões: (1) Quais os principais processos de Open Source Design? (2) Quais as principais etapas de cada processo? (3) Quais são os estágios de desenvolvimento que permitem a colaboração/participação do usuário? (4) Em qual ambiente (digital ou físico) acontece esta participação? (5) É necessário acesso a quais ferramentas: softwares (ambiente digital) ou equipamentos (ambiente físico)? (6) Os usuários de TA têm acesso às ferramentas de desenvolvimento de projeto? (7) Os usuários devem ter habilidades específicas ou as ferramentas de desenvolvimento são adaptadas para todos os usuários? (8) Como estes usuários podem ser beneficiados pelos projetos de design aberto?

As etapas de participação do usuário em processo de Design aberto foram comparadas com as etapas da abordagem do design centrado no humano e do design participativo e correlacionados estes termos e definições com o termo "assistive technology". 
Em cada etapa, foram analisados quatro fatores de influência sobre o nível de participação: os usuários, as habilidades do usuário, o ambiente de interação e as ferramentas (Figura 01).

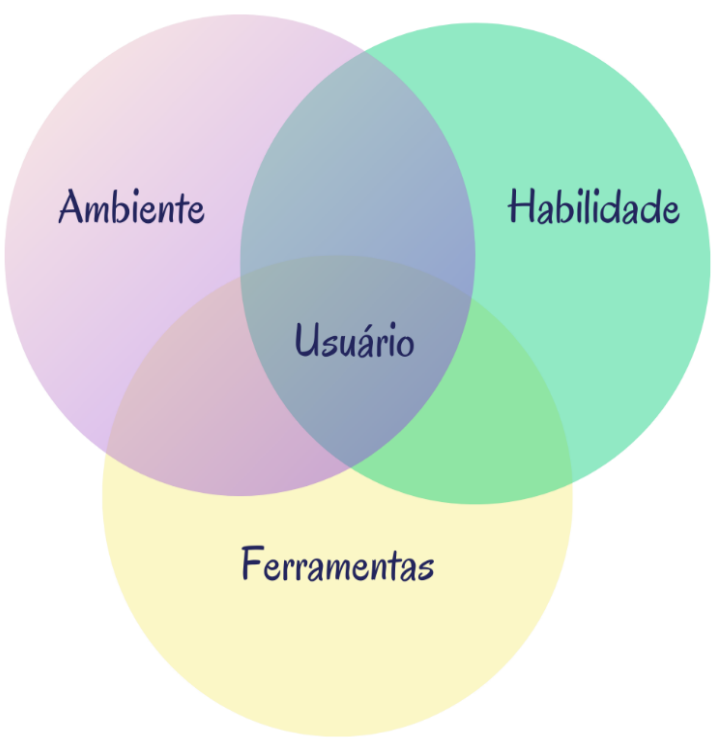

Figura 01. Aspectos que influenciam o grau de participação de usuários em processos de design. Fonte: Elaborado pelos autores (2021).

\section{Resultados e Discussões}

Na revisão sistemática foram encontrados os conceitos de abertura à participação de usuários em processos de desenvolvimento de produtos por Inovação Aberta (Open-Innovation), Design Participativo (Participatory Design) e Design centrado no humano (User centered Design). A Inovação aberta é uma prática especialmente desenvolvida dentro de empresas por meio de entradas e saídas propositais de conhecimento para acelerar a inovação interna e expansão dos mercados para uso externo da inovação. A prática incorpora o modelo de negócios como fonte de criação e captura de valor (CHESBROUGH; VANHAVERBEKE; WEST, 2006). Os autores Schuurman, (2015) e Leber, (2018), mostraram a participação dos usuários nas etapas iniciais ou finais. Deste modo, a Open Innovation não pertence à abordagem aberta, pois o compartilhamento de conhecimento fica limitado a uma estrutura contratual e sujeitas a acordos de não divulgação e não aberto livremente (MARAIS; SCHUTTE, 2009).

O Design participativo surgiu por meio de equipes interdisciplinares para atender e executar atividades projetuais cada vez mais complexas, com múltiplos requisitos e curto espaço de tempo (FONTANA; HEEMANN; FERREIRA, 2012). O processo perpassa todos os níveis de trabalho: estratégico (definição de problemas e objetivos), tático (abordam as metas pelas quais o objetivo deve ser alcançado) e operacional (o que de fato será realizado). Assim, o design participativo demanda um alto nível de confiança e comprometimento entre os envolvidos (HEEMANN; LIMA; CORREAA, 2010). Da mesma forma, o CoDesign, que apresenta uma abordagem participativa com possibilidade de comunicação entre os parceiros especialistas e não-especialistas além de possibilitar a intervenção, participação e envolvimento de todos os participantes, independentemente do seu conhecimento, prática profissional, social e cultural (ÁGUAS, 2012). Os processos colaborativos apresentam uma contribuição efetiva sobre o processo de design, consciência e tomadas de decisão. O estudo de Span, (2014) mostrou que a participação de usuários com 
demência no processo de desenvolvimento de uma ferramenta interativa para web fez com estes se sentissem mais envolvidos e compartilhassem mais informações sobre questões diárias do que antes.

Todos estes métodos, no entanto, são aplicados em diferentes etapas do desenvolvimento do projeto para usuários com diferentes habilidades. Sendo assim, pode-se observar que estes processos encontrados apresentam um cenário de participação dos usuários diferente do conceito original. A abertura de processos remonta às inovações tecnológicas que permitiram a criação de um ambiente digital e de conectividade da chamada "aldeia global" (MCLUHAN, 1964). Este processo iniciou com os desenvolvedores de softwares se adaptaram rapidamente e lideraram os processos de mudança. Foi assim com o conceito de Open Source (código aberto), cunhado em 1999 por Eric Raymond (RAYMOND, 1999). A comunidade de desenvolvedores, começou uma narrativa própria para se contrapor à lógica de mercado que privilegiava o modelo de desenvolvimento de software proprietário ou não-livre, exemplificado pela Microsoft ${ }^{\circledR}$. As forças básicas da organização industrial e direitos de propriedade intelectual começaram a serem discutidas, por meio da alegação de que o código-fonte, o principal ativo de conhecimento de uma empresa de software, é criado com mais eficiência em um cenário de colaboração entre desenvolvedores de forma descentralizada e em larga escala (WEBER, 2004). As práticas bem-sucedidas de contribuição aberta e democratização de produção de softwares, serviram de inspiração a outros meios, como por exemplo, a produção de recursos de comunicação e os projetos de objetos físicos, open source hardware (OSH), entre outros. (VAN ABEL et al., 2011; POWEL, 2012). Neste sentido, o OSH consiste em um artefato físico, elétrico ou mecânico, cujas informações de design são disponibilizadas para que qualquer pessoa faça, modifique, distribua e use (TAPR, 2007).

A implementação do conceito Open Source Software para o contexto de produtos físicos (Open Source Hardware) foi chamada de Design aberto (Open Design) (RAASCH; HERSTATT; BALKA, 2009). Para a mesma definição, autores como Fjeldsted, et al. (2012) e Zhang e Li (2017), Boousseau (2018) e Bonvoisin et al. (2018), usaram, de forma equivalente, os termos Open Source Development, Open Source Design, Open-X e Open Source Product Development. Para Bonvoisin et al. (2018), o Open Design consiste na abertura, ou seja, livre distribuição e uso de projetos, arquivos e ideias de produtos e processo de design transparente, executado de forma coletiva, permitindo a participação de todas as pessoas interessadas. Assim como o conceito original, Open Source Software, todas as etapas, devem ser acessíveis e (re)utilizáveis por qualquer pessoa e para qualquer finalidade (BOISSEAU; OMHOVER; BOUCHARD, 2018).

Assim, o Design aberto deve apresentar os três grandes estágios de design de forma aberta: (E1) entrada no processo (input) e definição do gap ou lacuna, que contempla o problema; (E2) desenvolvimento: atividades, com a utilização de diversos instrumentos, técnicas e métodos; e (E3) saída (output), produto.

Os processos encontrados de Inovação aberta, Design participativo e Design centrado no usuário, apresentam graus de abertura em algumas etapas e fechamento em outras. O design centrado no humano é um método tradicional, onde nem o estágio 2, nem o estágio 3 estão abertos (BOISSEAU; OMHOVER; BOUCHARD, 2018). No contexto de tecnologias assistivas (TA), Cook e Polgar (2008, p. 90) apontaram alguns pontos fundamentais para que um dispositivo possa vir a ser colaborativo: (1) parceria entre todos os envolvidos; (2) a solução de um problema deve ser compartilhada com todos; (3) respeito, disponibilização de conhecimento e contribuição; e (4) cada participante ter a possibilidade de obter as informações necessárias para realizar o seu papel. A Open Assistive Technology foi definida para incluir amplamente qualquer produto, dispositivo ou equipamento adquirido comercialmente, modificado ou personalizado para realizar algo que não seria possível de outra forma (ASSISTIVE TECH, 1998). A tecnologia abrange uma ampla gama de equipamentos, desde dispositivos simples de baixa tecnologia, como corrimãos e alças, até equipamentos de alta tecnologia, que incluem cadeiras de rodas elétricas e robôs. O conceito Open-Source Assistive Technology foi descrito para o desenvolvimento do primeiro repositório de software de código aberto dedicada à Tecnologia Assistiva (JUDGE et al., 2006). Com base na web, o repositório foi projetado para 
abrigar uma comunidade de médicos, profissionais de TA, usuários finais e desenvolvedores/programadores de código aberto. O projeto foi alterado e passou para Open Assistive.

No entanto, não são considerados Design Aberto, os projetos apresentando a saída aberta e disponibilizando as fontes para serem baixadas livremente e software gratuito, como modelos $2 \mathrm{D}$ com licença Creative Commons, que garantem uma infraestrutura técnica e jurídica para o compartilhamento de conteúdo. O fato é que o compartilhamento ao final de um projeto já desenvolvido de estágio (E3) não é considerado Design Aberto.

O nível de participação do usuário é que define se o Design é aberto ou não. De acordo com Damodaran (1996), há três níveis de participação dos usuários no processo de Design: (1) nível de participação varia entre usuário Informativo (UI), que fornece informações e (2) usuário consultivo (UC), que comenta sobre soluções de design e (3) usuário participativo (UP), que participa do processo e tomada de decisões relacionadas a todo o sistema. A participação está restrita a habilidades dos usuários, serem adquiridas com treinamentos ou estímulos: (1) habilidade técnica (HT): conhecimento, experiência de uso de produto ou serviço; (2) habilidade social (HS): devido ao comprometimento com o processo e relacionamento com outros participantes; (3) habilidade comportamental (HC): que indica busca de soluções, proatividade e está relacionada a tomada de decisões.

As ferramentas ou métodos de participação, refletem diretamente o grau de abertura do processo. No caso do Design centrado no usuário, que é um método de design tradicional e foca principalmente na compreensão dos usuários e no contexto de uso dos produtos, porém os usuários são meramente objetos examinados de fora. Os designers ou facilitadores escolhem o conjunto apropriado de métodos de participação do usuário em cada estudo. A maioria dos métodos permite uma comunicação presencial entre um pequeno grupo de participantes ou um feedback limitado de muitas pessoas, com a utilização de questionários online (MARKUS; MAO, 2004).

Os métodos utilizados são: questionários, entrevistas e pesquisas de opinião, que auxiliam o designer a obter dados interessantes para a sua investigação. Podem ser realizados também os grupos focais (focus group), que são grupos estruturados de discussão e liderados por um mediador (KARLSSON; KILDSGAARDS, 2012).

As entrevistas contextuais são usadas para identificar as reais necessidades dos usuários, diários de usuário e sondas culturais (cultural probes), onde os cidadãos são solicitados a contar histórias e compartilhar observações, como texto e imagens sobre um determinado tópico, a vida cotidiana na área, sonhos futuros (GAVER; DUNNE; PACENTI, 1999) e o Brainstorming (tempestade de ideias), onde os participantes devem falar ou escrever o maior número de ideias em um período (FRIEDRICH, 2013).

A etapa de prototipagem, de papel, auxilia a visualizar e a testar ideias e conceitos em pouco tempo e com baixo custo. No workshop, um palestrante apresenta o conteúdo, fomenta a reflexão por meio do grupo e aplica a uma atividade prática que pode ser realizada num estudo de campo (KARLSSON;

KILDSGAARDS, 2012). Martin (2012), mostrou a participação de usuários os estágios iniciais de desenvolvimento de dispositivos médicos. A consulta inicial identificou uma necessidade clínica para o dispositivo, as populações-alvo foram definidas e seus requisitos coletados.

O Design participativo e o Co-Design podem usar os métodos tradicionais, porém podem ser mais acessíveis em abordagens abertas utilizando as ferramentas online e as mídias sociais. Concurso de ideais (Idea competition) são normalmente campanhas online, durante as quais qualquer pessoa pode sugerir uma ideias para um tópico definido (ARNOLD; BARTH, 2012). Os storyboards contam uma história visual de um serviço ou solução planejada, por exemplo na forma de uma história em quadrinhos. De forma mais avançada, filmes podem ser usados para avaliar soluções online. 
Mock-ups são protótipos leves que ilustram certos aspectos da solução como um objeto tangível. Estudo de campo avaliam a adaptação de um usuário e uma solução por um período (FRIEDRICH, 2013). Na participação do cidadão (Citizen participation), a tomada de decisão pode ser organizada por votação, durante as reuniões e fóruns de diálogo (reunião de diálogo focada), em que os cidadãos podem deixar suas ideias na forma de videoclipe, imagem ou texto; painéis do usuário (user panels) em que o grupo fornece sugestões e comentários sobre um determinado serviço ou problema por SMS ou email; ou ferramenta online, como chat e $e$-voting (votação eletrônica). O líder do projeto pode enviar perguntas por SMS ou email (KARLSSON; KILDSGAARDS, 2012).

As mídias sociais fornecem ferramentas simples para colaboração, dando a todos uma nova via para a participação e a co-criação de conteúdo, como blog, chat, videoconferência e mensagens. As discussões online podem ocorrer em tempo real após uma reunião presencial. Existem plataformas com laboratórios web pagos e participativos, usados para projetar produtos e serviços de mídia digital, por exemplo: Open Web Lab e Owela, criado visando alcançar o conceito de Open User-centred Design (FRIEDRICH, 2013).

As Figuras 2, 3 e 4 mostram a abertura dos principais estágios de desenvolvimentos dos processos de design DHC, DP e DA.

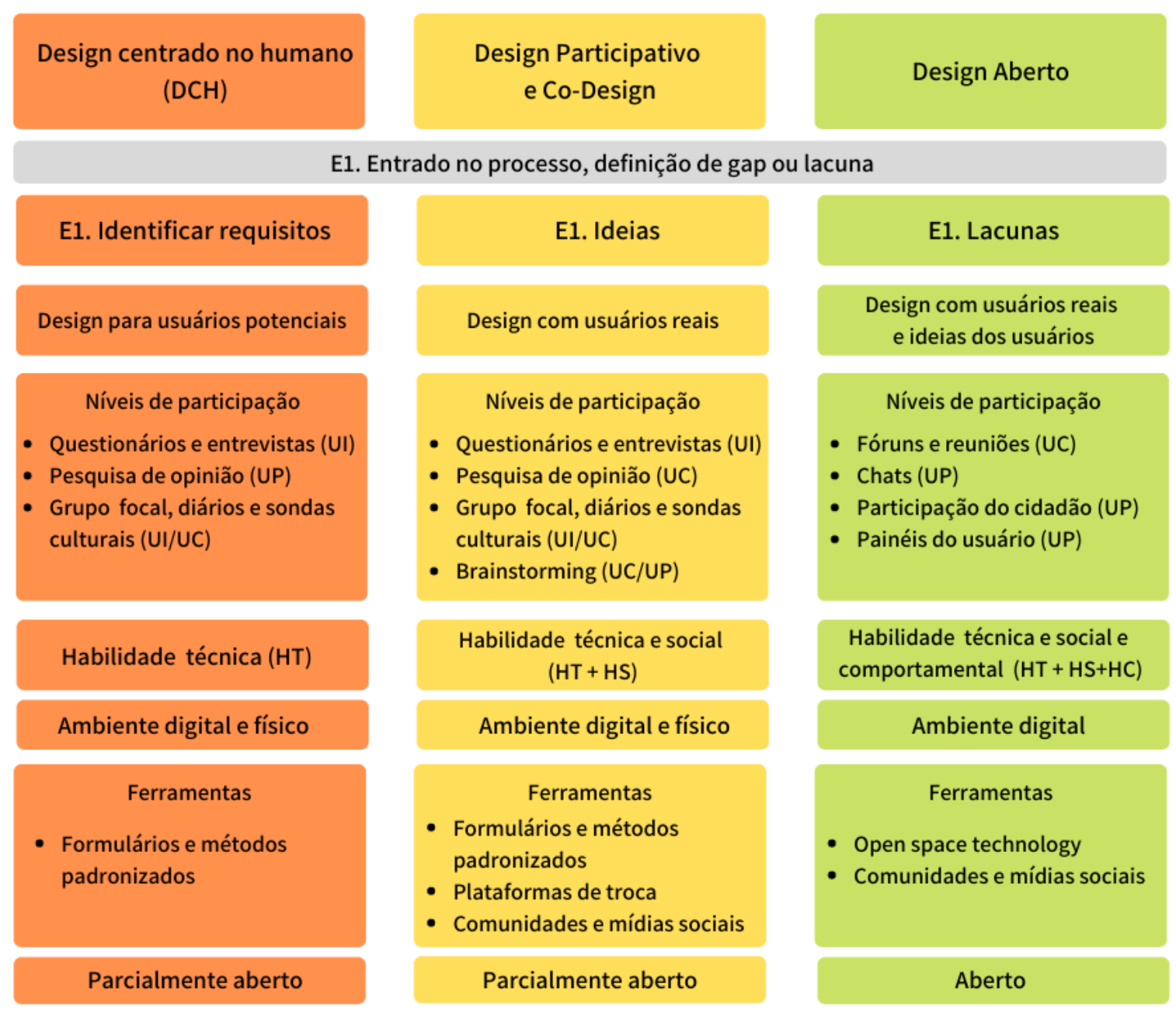

Figura 02. Etapa de entrada e definição do gap ou lacuna Fonte: Elaborado pelos autores (2021). 


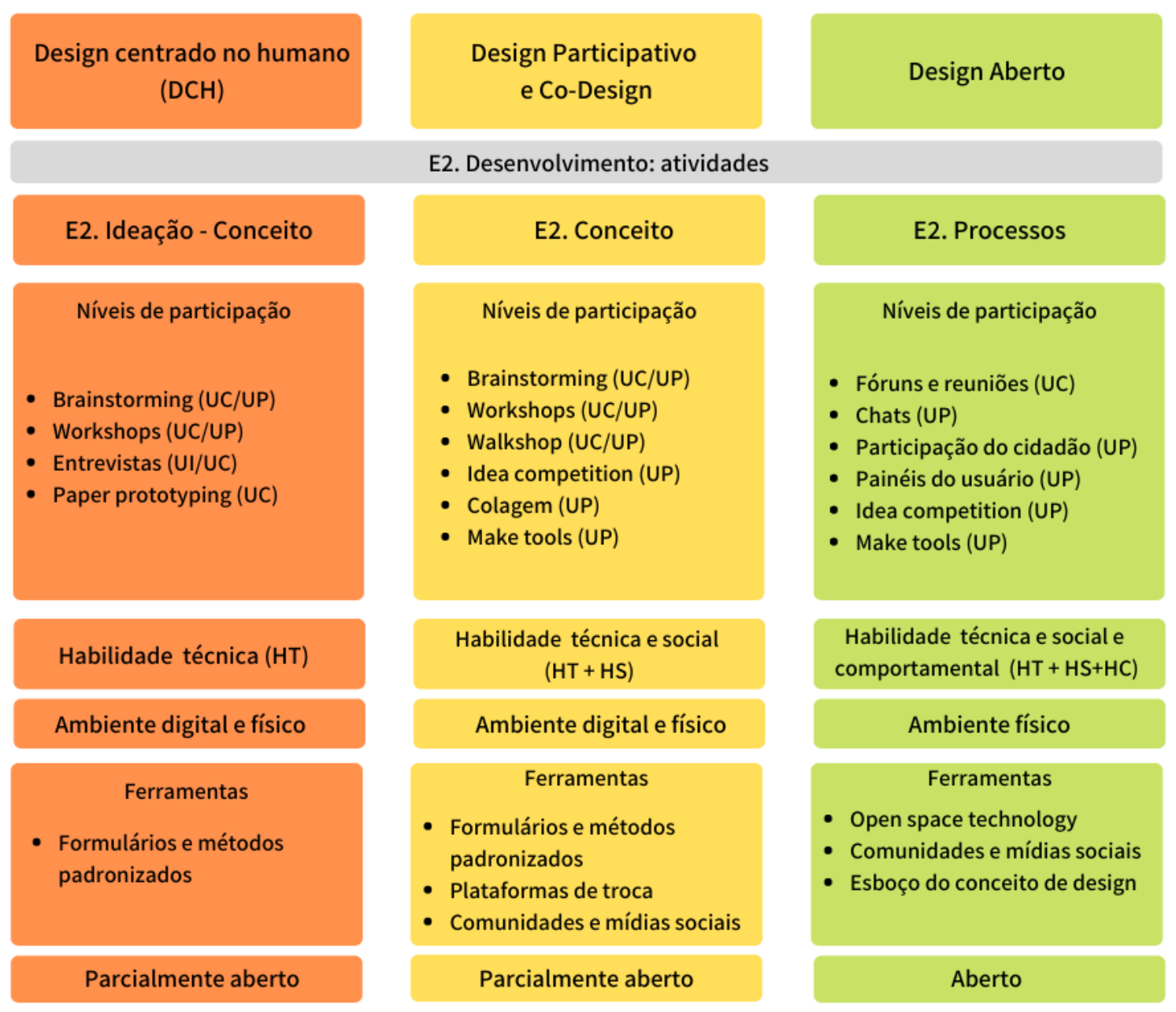

Figura 03. Etapa de desenvolvimento e atividades

Fonte: Elaborado pelos autores (2021).

Na plataforma, os usuários são convidados a participar de reuniões online no conceito de ambiente aberto para produção coletiva, por meio de tópicos (postagens) e direcionados pelos mediadores que avaliam os comentários relativos. É um espaço para colocar as ideais, fazer brainstorming, avaliar cenários de futuros produtos, comentá-los e testá-los num laboratório online. Existem outras modalidades de plataformas, como o ESA, que permite gratuitamente o envio de ideias para tecnologia e aplicativos espaciais. A partir de uma triagem, o projeto pode ficar fechado ao público e passar a receber financiamento privado. Há diversos softwares disponíveis para a geração de laboratórios de pesquisa de usuários online (research lab) gratuitos, de forma dispersa, alguns para teste de usabilidade ou reuniões, como: Open Broadcaster Software (OBS) e Open Virtual Usability Lab (OpenVULab). A Open Space Technology (OST) é uma abordagem para encontros de grupos que difere da maioria das ferramentas de facilitação e diálogo, porque são os próprios participantes que definem o que vai ser conversado dentro do contexto apresentado.

As ferramentas de comunicação síncrona online podem ser pagas ou gratuitas por videoconferência (Zoom; Skype, entre outras), Voip (Voice over Internet Protocol), wikis, chats, entre outros.

Muitas destas novas abordagens se relacionam ao contexto do Open Design, onde a comunidade (designers e usuários) começa a inovar, a partir de ferramentas baseadas na web (FRIEDRICH, 2013; OWEN, 1997). 


\begin{tabular}{|c|c|c|}
\hline $\begin{array}{l}\text { Design centrado no humano } \\
\text { (DCH) }\end{array}$ & $\begin{array}{c}\text { Design Participativo } \\
\text { e Co-Design }\end{array}$ & Design Aberto \\
\hline \multicolumn{3}{|c|}{ E3. Saída (output): produto } \\
\hline $\begin{array}{c}\text { E3. Construir protótipos } \\
\text { testáveis }\end{array}$ & E3. Prototipagem & E3. Prototipagem \\
\hline $\begin{array}{l}\text { Níveis de participação } \\
\text { - Prototipagem (UC) } \\
\text { - Teste de usabilidade (UI) } \\
\text { - Estudo de campo (UI/UC) } \\
\text { - Questionário de satisfação } \\
\text { (UI/UC) }\end{array}$ & 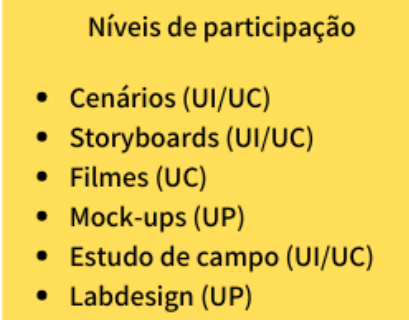 & $\begin{array}{l}\quad \text { Níveis de participação } \\
\text { - Open space technology (UP) } \\
\text { - Comunidades (UP) } \\
\text { - Labdesign, softwares livres, } \\
\text { desenho técnico, modelagem } \\
\text { 3D e arquivos CAM (UP) }\end{array}$ \\
\hline Habilidade técnica (HT) & $\begin{array}{l}\text { Habilidade técnica e social } \\
\qquad(\mathrm{HT}+\mathrm{HS})\end{array}$ & $\begin{array}{l}\text { Habilidade técnica e social e } \\
\text { comportamental (HT + HS+HC) }\end{array}$ \\
\hline Ambiente digital e físico & Ambiente digital e físico & Ambiente físico \\
\hline $\begin{array}{l}\text { - Formulários e métodos } \\
\text { padronizados }\end{array}$ & $\begin{array}{l}\text { Ferramentas } \\
\text { - Formulários e métodos } \\
\text { padronizados } \\
\text { - Repositórios/arquivos CAD e } \\
\text { CAM }\end{array}$ & $\begin{array}{l}\text { Ferramentas } \\
\text { - Planilha de gerenciamento de } \\
\text { projetos (lista de materiais) } \\
\text { - Esquemas, fotos e vídeos } \\
\text { - Repositórios/arquivos CAD, } \\
\text { CAM e CAE }\end{array}$ \\
\hline Parcialmente aberto & Parcialmente aberto & Aberto \\
\hline
\end{tabular}

Figura 04. Etapa de saída - produto

Fonte: Elaborado pelos autores (2021).

Fazem parte deste novo paradigma de ambiente aberto, os laboratórios de Design. Os laboratórios de usabilidade são utilizados para testar a usabilidade de produtos ou serviços em ambiente controlado. De maneira bem mais ampla, o laboratório de Design é um espaço colaborativo de exploração projetista, que usa a experimentação, tal como oficina, estúdio ou ateliê, no contexto de uso real do produto e com autoria compartilhada (BINDER; BRANDT, 2008). A digitalização destes ambientes, segundo Boisseau, Omhover e Bouchard (2018), está diretamente relacionada a disponibilização de desenhos auxiliados por computador (CAD), Manufatura (CAM) e Engenharia (CAE). A digitalização facilita a troca de objetos de contorno (boundary objects), que são críticos em um processo de design colaborativo.

Como apresenta a Figura 05, pode-se perceber que o nível de abertura de um processo de design, está diretamente relacionado a participação do usuário em diferentes estágios dos processos (UCD, PD e CO e Open Design). A etapa 3, saída do DCU é fechada, ou seja, o produto ou serviço resultante do processo não está disponível gratuitamente para os usuários ou sob uma licença aberta. A participação do usuário em todo o processo foi de UC e UI. O PD e CD apresentam a participação do UC nas etapas 1 e 3, e UP na etapa 2. Interessante notar que a disponibilização do produto ou serviço resultante, como modelos 3D disponíveis 
para impressão, e a disponibilização da documentação completa do passo a passo de fabricação, tutoriais de uso, lista de materiais, fotos e vídeos, não é suficiente para deixar o processo Open Design.

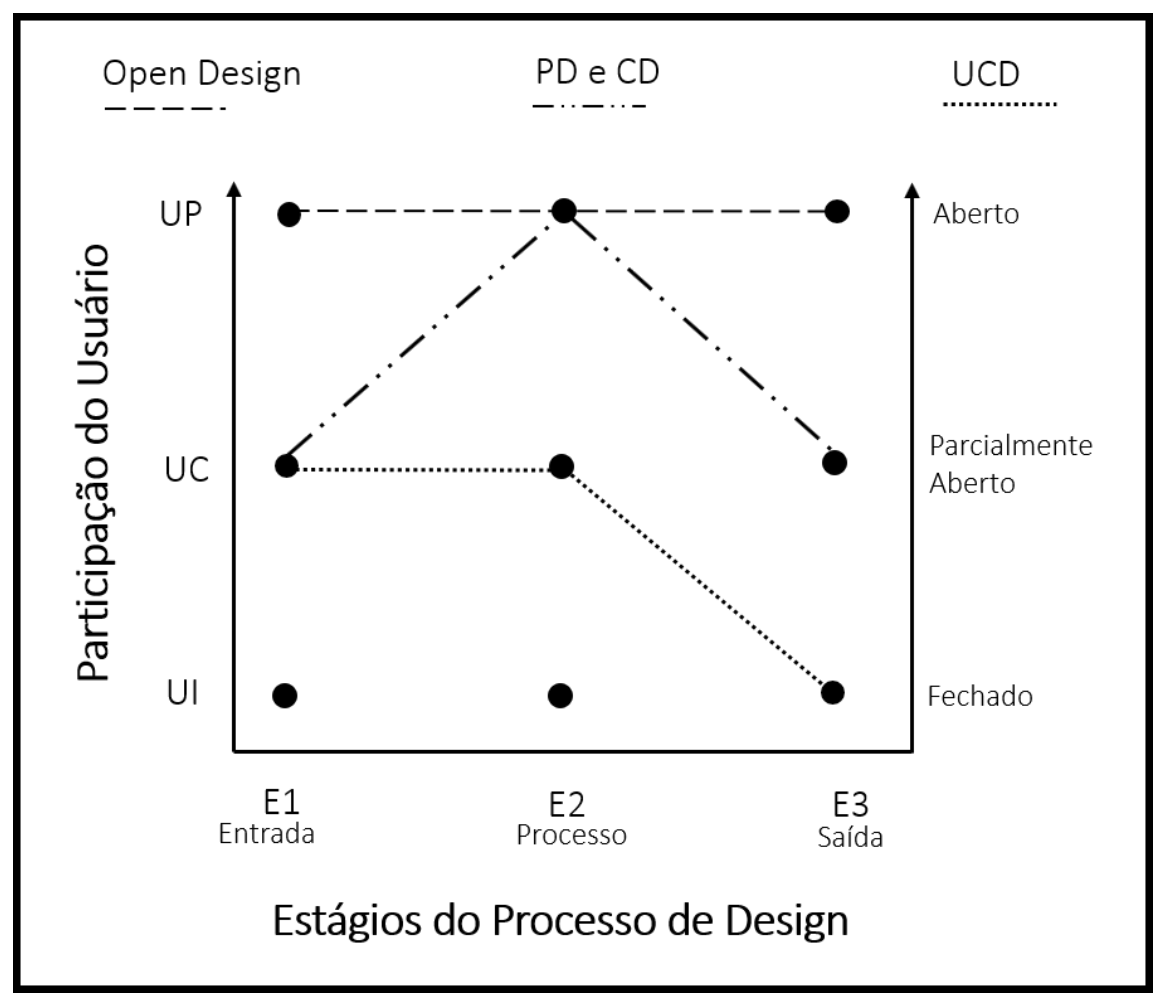

Figura 05. Níveis de participação dos usuários em relação às etapas dos diferentes métodos de Design: Usuário consultivo (UC), usuário informativo (UI) e usuário participativo (UP). Fonte: Elaborado pelos autores (2021).

A participação dos usuários em todas as etapas é uma condição para o processo aberto. Porém, as habilidades requeridas mudam, conforme os processos tornam-se abertos. Sendo que, as habilidades sociais e de comunicação tornam-se mais importantes em processos abertos. No geral, as habilidades sociais auxiliam os indivíduos a tomar decisões, interagir por comunicação verbal ou não verbal, aprender as convenções sociais ou regular as emoções (BOUCK; LONG, 2020). Observa-se assim, uma oportunidade de desenvolvimento de ferramentas e boundary objects voltadas as habilidades sociais e comportamentais de usuários de TA. O ambiente digital permite um alto controle das atividades, por exemplo, na organização, no tempo de permanência em uma tarefa e nos estímulos para atrair a atenção. No entanto, a navegação em ambiente digital, pode ser uma barreira devido a aquisição de dispositivos tecnológicos, a necessidade de treinamento ou mediação especializada.

\section{Conclusões}

Por meio de revisão sistemática foram encontrados os conceitos de código aberto, Design participativo e Design centrado no usuário, bem como suas demais nomenclaturas e variações. Os recursos Open Source estão cada vez mais disponíveis, no entanto, a disseminação de ferramentas para desenvolvimento de projetos de design e a abertura de processos de fabricação de novos dispositivos físicos e digitais precisa incluir os usuários de TA. Estes não devem desenvolver funções meramente informativas, no estágio de saída dos projetos disponibilizados prontos para download. As ferramentas que promovem o usuário 
participativo, ou de mídias sociais e que se adaptam as habilidades dos usuários, podem promover o maior engajamento de usuários no desenvolvimento de produtos de TA. Portanto, as ferramentas e não as habilidades dos usuários, devem promover maior participação nos processos de design. A participação dos usuários em todas as etapas é uma condição para que o processo seja considerado aberto, sendo que as habilidades sociais e de comunicação são as mais importantes. O foco no desenvolvimento de comunidades de suporte online e softwares com interfaces mais amigáveis e menos especializadas, maior disponibilidade de pacotes gratuitos ou de código aberto podem ser aplicados neste sentido. Como visto, os processos de inovação aberta são desenvolvidos sob perspectiva de sigilo industrial, mais voltados aos fins lucrativos. O processo de design participativo está mais próximo ao ideal do design aberto. A comparação dos processos novos e tradicionais, mostrou que o estágio 3, do Design centrado no usuário é o mais restritivo, onde o usuário realiza papel informativo e consultivo, mas não tem poder de decisão. O processo de abertura não trata da capacitação para a utilização de ferramentas atuais, mas na transformação das ferramentas atuais visando a utilização de todos. Neste sentido, existem diversas oportunidades para a produção e compartilhamento de TA. Para tanto, será necessário buscar formas de vencer as barreiras tecnológicas e alcançar uma comunicação online adequada aos perfis dos usuários de TA.

\section{Referências Bibliográficas}

ARIAS, V.; HURST, A.; PERR, A. Designing a remote framework to create custom assistive technologies. In: International ACM SIGACCRSS, Conference on computer and accessibility, 22, Proccedingas [...], 2021. DOI: https://doi.org/10.1145/3373625.3418022

ÁGUAS, S. Do design ao co-design: Uma oportunidade de design participativo na transformação do espaço público. Revista Científiques de la Universitat de Barcelona, Barcelona, n. 22, p. 57-70, 2012.

ARNOLD, M.; BARTH, V. Open innovation in urban energy systems. Energy Efficiency, v. 05, p. 351364, 2012.

ASSISTIVE TECH ACT. Section 3, 1998. Disponível em: https://www.section508.gov/tools/playbooks/technology-accessibility-playbook-intro. Acesso em: 05 mar. 2020.

BERSCH, R. Introdução à Tecnologia Assistiva. Porto Alegre, 2013.

BINDER, T.; BRANDT, E. The Design: Lab as platform in participatory design research. CoDesign, v. 4, n. 2, p. 115-129, 2008. DOI: https://doi.org/10.1080/15710880802117113

BOISSEAU, É.; OMHOVER, J. F.; BOUCHARD, C. Open design: A state of the art review. Design Science, v. 4, n.3, 2018. DOI: https://doi.org/10.1017/dsj.2017.25

BONVOISIN, J.; BUCHERT, T.; PREIDEL, M.; STARK, R. G. How participative is open source hardware? Insights from online repository mining. Design Science, v.4, n.19, 2018. DOI:

https://doi.org/10.1017/dsj.2018.15

BOUCK, E.C; LONG, H. Assistive Technology for Students with Disabilities: An Updated Snapshot. Journal of Special Education Technology, 2020. Doi:

http://doi.org/10.1177/0162643420914624

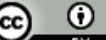

Revista Ergodesign \& $\mathrm{HCl}$

ISSN 2317-8876 
CHAVES, I. G. O Design centrado no humano conectado e colaborativo. 2019. 314p. Tese (Doutorado) Universidade de São Paulo, Faculdade de Arquitetura e Urbanismo, São Paulo, 2019. Disponível em: https://teses.usp.br/teses/disponiveis/16/16134/tde-19092019-163604/en.php. Acesso em 15 jun 2021.

CHESBROUGH, H.; VANHAVERBEKE, W.; WEST, J. Open Innovation: Researching a New Paradigm: Oxford University Press, Oxford, 2006.

COOK, A. M.; POLGAR, J. M. Cook \& Hussey's Assistive Technologies: Principles and Practice, 3rd ed., Philadelphia: Elsevier Inc., 2008.

DAMODARAN, L. User involvement in the systems design process: a practical guide for users. Behaviour \& Information Technology, v. 15, n. 16, p. 363-377, 1996.

FJELDSTED, A. S. et al. Open Source Development of Tangible Products-from a business perspective. In: NORD DESIGN, 9th, 2012, Aalborg, Dinamarca. Proceedings... Aalborg: 2012.

FONTANA, I. M.; HEEMANN, A.; FERREIRA, M. G. G. Design colaborativo: fatores críticos para o sucesso do co-design. In: Congresso Sul-Americano de Design de Inovação, 4, 2012, São Paulo. Anais [...]... São Paulo: 2012. p. 371-382.

FRIEDRICH, P. Web-based co-design: Social media tools to enhance user-centred design and innovation processes. Doctoral dissertation. VTT Science 34: Espoo, Finland. 2013, 185p. Disponível em: https://www.vttresearch.com/sites/default/files/pdf/science/2013/S34.pdf. Acesso em: 14 abr 2020.

GAVER, B.; DUNNE, T.; PACENTI, E. Cultural Probes. Interactions, v. 06, n. 01, p. 21-29, 1999. DOI: https://doi.org/10.1145/291224.291235

GEORGES, A.; SCHUURMAN, B.; D., BACCARNE, B. AND COOREVITS, L. User engagement in living lab field trials, Info, v. 17,n. 4, p. 26-39, 2015. DOI: https://doi.org/10.1108/info-01-2015-0011

HAMIDI, F., et al. Do-It-Yourself (DIY) assistive technology: A communication board case study. In: Miesenberger K., Fels D., Archambault D., Peňáz P., Zagler W. (eds) Computers Helping People with Special Needs. ICCHP 2014. Lecture Notes in Computer Science, vol 8548. Springer, Cham

HEEMANN, A.; LIMA, P. J. V.; CORRÊA, J. S. Fundamentos para o Alcance da Colaboração em Design. Design e Tecnologia, v. 18, n. 02, p. 1338-1349, 2014.

HURST, A.; KANE, S. Making "making" accessible. In: INTERNATIONAL CONFERENCE ON INTERACTION DESIGN AND CHILDREN, 13, 2013, New York. Proceedings [...] New York: 2013, p. 635-638.

INTERACTION DESIGN FOUNDATION. Disponível em: https://www.interaction-design.org. Acesso em 25 jun 2021.

INTERNATIONAL ORGANIZATION OF STANDARDIZATION. ISO 9241-210 - Ergonomics of human-system interaction - part 210: Human Centered Design for Interactive Systems, 2010

JUDGE, S.; LYSLEY, A.; WALSH, J.; JUDSON, A.; DRUCE, S. OATS - Open source assistive technology software: a way forward. Communication Matters Journal, v.19, n.3, p. 11-12, 2006. 
KARLSSON, A.; KILDSGAARD, I. Multi-stakeholder dialogue on green urban climate adaptation. In: Swedish Environmental Research Institute, IVL, 2021. Disponível em: https://www.ivl.se/download/18.34244ba71728fcb3f3f82f/1591704972529/B2107.pdf. Acesso em 25 jun 2021.

LANUTTI, J. N. L.; MEDOLA, F. O.; GONÇALVES, D. D.; SILVA, L. M.; NICHOLL, A. R. J. PASCHOARELLI, L. C. The significance of manual wheelchairs: a comparative study on male and female users. In: International Conference on Applied Human Factors and Ergonomics and the Affiliated Conferences, 6., 2015, Las Vegas. Proceedings [...]: Proceeding Manufacturing, v.03, p.6079-6085, 2015. DOI: http://doi.org/10.1016/j.promfg.2015.07.752

LEBER, M.; IVANISEVI'C, A.; BOROCKI, J.; RADISIC, M.; SLUSARCZYK, B. Fostering Alliances with Customers for the 'Sustainable Product Creation. Sustainability, 2018, v.10, 3204.

MARAIS, S. J.; SCHUTTE, C. S. L. The development of open innovation models to assist the innovation process. In: SOUTHERN AFRICAN INSTITUTE FOR INDUSTRIAL ENGINEERING, 2009.

Anais...Southern African: 2009. p. 96-116.

MARKUS, M.; MAO, Y. User Participation in Development and Implementation: Updating an Old Tired Concept for Today's IS Contexts. Journal of the Association for Information Systems, v. 05, n.11-12, p. 514-544, 2004.

MARTIN, J.L.; CLARK, D. J.; MORGAN,S.P., CROWE,J.A.; MURPHY,E. A user-centred approach to requirements elicitation in medical device development: A case study from an industry perspective, Applied Ergonomics, v. 43, Issue 1, p.184-190, 2012. DOI: https://doi.org/10.1016/j.apergo.2011.05.002.

McDONAGH, D. et al. User centered approach to the supra-functional needs of people living with amyotrophic lateral sclerosis (ALS). In: Design of Medical Devices Conference, DMD 2021, Proceedings [...] Mineápolis, 2021.

McLUHAN, M. Understanding Media: The Extensions of Man. New York: McGraw Hill, 1964. OWEN, H. Open space technology: A user's guide. 3nd ed. New York: Berrett-Koehler, 2008.

PEZZINI, M.; SCHULENBURG, R.; ELY, V. H. M. B. Toolkit de design centrado no humano para o mini morar. Design \& Tecnologia, v. 15, p. 24-50, 2018.

POWELL, A. Democratizing production through open source knowledge: From open software to open hardware. Media Culture \& Society, v.34, n.6, p.691-708. 2012.

RAASCH, C.; HERSTATT, C.; BALKA, K. On the open design of tangible goods. RD Manag. v.39, p.382393, 2009. DOI: https://doi.org/10.1111/j.1467-9310.2009.00567.x

RAYMOND, E. S. The cathedral and the bazaar. Knowledge, Technology \& Policy, v. 12, n. 03, p. 23-49, 1999. DOI: http://doi.org/10.1007/s12130-999-1026-0

STAR, S. L.; GRIESEMER, J.R. Institutional ecology, translations and boundary objects: Amateurs and professional in Berkeley's Museum of Vertebrate Zoology, 1907-39. Social studies of science, v.19, n.3, p.387-420, 1989.

SOARES, J. M. M. et al. Multicase study on product design in the area of assistive technology in Brazil. Disability and Rehabilitation: Assistive Technology, v. 15, n. 04, p. 442-452, 2019. 
SPAN, M., SMITS, C., GROEN-VAN DE VEN, L. M., CREMERS, A., JUKEMA, J., VERNOOIJDASSEN, M. An interactive web tool to facilitate shared decision making in dementia: design issues perceived by caregivers and patients. International Journal of Advances in Life Science and Technology. 6, 107-121, 2014

TAPR OPEM HARDWARE LICENSE VERSION 1.0. Web TAPR org, 2007. Disponível em: http://www.tapr.org/TAPR Open_Hardware License v1.0.txt. Acesso em: 08 abr. 2020.

WEBER, S. Open source software in developing economies, 2003. Disponível em:

http://www.ssrc.org/programs/itic/publications/ITST_materials/webernote2.pdf . Acesso em 05 set de 2020.

VAN ABEL, B. et al. Open Design Now: Why Design Cannot Remain Exclusive. Amsterdam: BIS Publishers, 256p., 2015.

ZHANG, S.; LI, Y. Modeling and Simulation Study of Designers Bidirectional Behavior of Task Selection in Open Source Design Process. Mathematical Problems in Engineering, 2017. DOI: http://doi.org/10.115 5/2017/6738139

\section{Agradecimentos}

Os autores deste artigo agradecem ao CNPq, FAPESC, CAPES, ao Programa de Pós-Graduação em Design da UDESC pelo apoio na realização da pesquisa. 